\title{
Antineutrophil cytoplasmic autoantibodies specific for myeloperoxidase cause glomerulonephritis and vasculitis in mice
}

\author{
Hong Xiao, ${ }^{1}$ Peter Heeringa, ${ }^{2}$ Peiqi Hu, ${ }^{3}$ Zhi Liu, ${ }^{3}$ Minglang Zhao, ${ }^{3}$ Yasuaki Aratani, ${ }^{4}$ \\ Nobuyo Maeda, ${ }^{1}$ Ronald J. Falk, ${ }^{5}$ and J. Charles Jennette ${ }^{1}$ \\ ${ }^{1}$ Department of Pathology and Laboratory Medicine, University of North Carolina at Chapel Hill, \\ Chapel Hill, North Carolina, USA \\ ${ }^{2}$ Department of Clinical and Experimental Immunology, University of Maastricht, Maastricht, The Netherlands \\ ${ }^{3}$ Department of Dermatology, University of North Carolina at Chapel Hill, Chapel Hill, North Carolina, USA \\ ${ }^{4}$ Kihara Institute for Biological Research, Yokohama City University, Totsuka, Yokohama, Japan \\ ${ }^{5}$ Department of Medicine, University of North Carolina at Chapel Hill, Chapel Hill, North Carolina, USA
}

\begin{abstract}
Antineutrophil cytoplasmic autoantibodies (ANCAs) are identified in the circulation of approximately $80 \%$ of patients with pauci-immune necrotizing and crescentic glomerulonephritis and systemic small vessel vasculitis, such as microscopic polyangiitis and Wegener granulomatosis. The most common antigen target for ANCAs is myeloperoxidase (MPO), which is found in neutrophils and monocytes. We report definitive experimental animal evidence that ANCAs are pathogenic. MPO knockout $\left(\mathrm{Mpo}^{-/-}\right)$mice were immunized with mouse MPO. Splenocytes from these mice or from control mice were injected intravenously into recombinase-activating gene-2-deficient (Rag2-/-) mice, which lack functioning B lymphocytes and $\mathrm{T}$ lymphocytes. All mice that received splenocytes developed mild to moderate glomerular immune deposits, but only mice that received $1 \times 10^{8}$ or $5 \times 10^{7}$ anti-MPO splenocytes developed severe necrotizing and crescentic glomerulonephritis, granulomatous inflammation, and systemic necrotizing vasculitis, including necrotizing arteritis and hemorrhagic pulmonary capillaritis. To test the pathogenic potential of antibodies alone, purified antiMPO IgG or control IgG was injected intravenously into Rag2 $2^{-/-}$mice and wild-type mice. Mice that received anti-MPO IgG but not mice that received control IgG developed focal necrotizing and crescentic glomerulonephritis with a paucity of glomerular Ig deposition. Thus, anti-MPO IgG alone was able to cause pauci-immune glomerular necrosis and crescent formation in the absence of function-

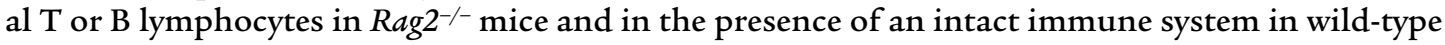
C57BL/6J mice. This animal model offers strong support for a direct pathogenic role for ANCA IgG in human glomerulonephritis and vasculitis.
\end{abstract}

J. Clin. Invest. 110:955-963 (2002). doi:10.1172/JCI200215918.

\section{Introduction}

The most common form of crescentic glomerulonephritis and the most common type of necrotizing systemic vasculitis in adults are associated with circulating antineutrophil cytoplasmic autoantibodies (ANCAs) $(1,2)$. ANCAs are specific for antigens in the primary granules of neutrophils and the peroxidasepositive lysosomes of monocytes. The two major anti-

Received for publication May 13, 2002, and accepted in revised form August 6, 2002.

Address correspondence to: J. Charles Jennette, Department of Pathology and Laboratory Medicine, 303 Brinkhous-Bullitt Building, University of North Carolina, Chapel Hill, Chapel Hill, North Carolina 27599-7525, USA. Phone: (919) 966-2421; Fax: (919) 966-4542; E-mail: jcj@med.unc.edu.

Conflict of interest: No conflict of interest has been declared.

Nonstandard abbreviations used: antineutrophil cytoplasmic autoantibody (ANCA); myeloperoxidase (MPO); proteinase 3 (PR3); recombinase-activating gene-2 (Rag2); wild-type (WT); immunofluorescence microscopy assay (IFA); blood urea nitrogen (BUN); hematoxylin and eosin (H\&E); C57BL/6J (B6) gen specificities are for myeloperoxidase (MPO-ANCA) (3) and proteinase 3 (PR3-ANCA) (4).

Numerous in vitro observations provide strong evidence that both MPO-ANCA and PR3-ANCA are directly involved in causing the glomerular and vascular inflammation of ANCA-associated glomerulonephritis and vasculitis $(5,6)$. For example, ANCA IgG stimulates cytokine-primed neutrophils and monocytes to undergo respiratory burst, release toxic and lytic granule constituents, adhere to endothelial cells, and kill endothelial cells $(5,6)$.

Until now, however, in vivo experimental animal observations have not offered definitive evidence for a pathogenic role for ANCAs (7). The experiments described in this article provide compelling evidence that ANCAs are directly pathogenic. These experiments document the induction of glomerulonephritis and vasculitis by the adoptive transfer of mouse anti-MPO splenocytes into immune-deficient mice or the passive infusion of mouse anti-MPO IgG into both immune-deficient and im- 
mune-competent mice. The resulting necrotizing and crescentic glomerulonephritis, pulmonary hemorrhagic capillaritis, and systemic necrotizing arteritis have remarkable pathologic similarity to human ANCA-associated glomerulonephritis and vasculitis.

\section{Methods}

Purification of mouse MPO. Mouse MPO was purified from WEHI-3 cells (a murine myeloid cell line purchased from American Type Culture Collection, Manassas, Virginia, USA) using a modification of the method of Hope et al. (8). Briefly, WEHI-3 cells were grown in McCOY5A medium with $10 \%$ FCS. Once the cells reached a density of $1.5 \times 10^{6}$ cells per milliliter, they were harvested by centrifugation and resuspended in buffer $\mathrm{A}$ (6.7 mM sodium phosphate, $\mathrm{pH} 6.0$; $1 \mathrm{mM} \mathrm{MgCl}_{2} ; 3 \mathrm{mM} \mathrm{NaCl} ; 0.5 \mathrm{mM}$ PMSF) at a ratio of $10 \mathrm{ml}$ of buffer to $1 \mathrm{ml}$ of cell pellet. The cells were lysed by Dounce homogenization on ice and then centrifuged at 20,000 $\mathrm{g}$ for 30 minutes. The pellets were resuspended in buffer A. Cetyltrimethylammonium bromide was added to a final concentration of $1 \%$, and the mixture was stirred vigorously for 2 hours at $4^{\circ} \mathrm{C}$. The insoluble material was removed by centrifugation at $20,000 \mathrm{~g}$ for 20 minutes at $4^{\circ} \mathrm{C}$. The solubilized material was dialyzed against buffer $\mathrm{B}(100 \mathrm{mM}$ sodium acetate, $\mathrm{pH} 6.3 ; 100 \mathrm{mM} \mathrm{NaCl}$ ) for 5 hours at $4{ }^{\circ} \mathrm{C}$. $\mathrm{CaCl}_{2}, \mathrm{MgCl}_{2}$, and $\mathrm{MnCl}_{2}$ were then added to a final concentration of $1 \mathrm{mM}$ each. The material was mixed end-over-end with $5 \mathrm{ml}$ of concanavalin A-Sepharose (Amersham Pharmacia Biotech, Piscataway, New Jersey, USA) overnight at $4^{\circ} \mathrm{C}$. The resin was poured into a Bio-Rad Econo-Column (Bio-Rad Laboratories, Hercules, California, USA). The MPO was eluted from the concanavalin A-Sepharose with 3-ml pulses of $750 \mathrm{mM}$ methyl $\alpha$-D-mannopyranoside in buffer B plus $1 \mathrm{mM}$ $\mathrm{CaCl}_{2}, \mathrm{MgCl}_{2}$, and $\mathrm{MnCl}_{2}$. The MPO-containing fractions were determined both by the green color and by A 430 values and were dialyzed against buffer C $(25 \mathrm{mM}$ sodium acetate, $\mathrm{pH} 8.5 ; 100 \mathrm{mM} \mathrm{NaCl}$ ) overnight at $4^{\circ} \mathrm{C}$. The sample was loaded onto a cation exchange column (HiTrap SP Sepharose HP [Amersham Pharmacia Biotech]; $5 \mathrm{ml}$ ) and eluted with $1 \mathrm{M} \mathrm{NaCl}(\mathrm{pH}$ 8.5). The eluate was loaded onto Superose 12 column (Amersham Pharmacia Biotech) $(60 \times 1.5 \mathrm{~cm})$ and eluted in buffer $\mathrm{C}$. The isolated MPO was dialyzed against water and concentrated with Centriprep (Millipore Corp., Bedford, Massachusetts, USA) at 2,000 g. The purity of the isolated MPO was confirmed by SDSPAGE and Western blot.

Mice. Mice lacking MPO ( $\mathrm{Mpo}^{-/-}$mice) were the sixthgeneration progeny of a backcross into $\mathrm{C} 57 \mathrm{BL} / 6 \mathrm{~J}$ mice (B6 mice) originally generated by Aratani et al. (9). Mice were genotyped using PCR-amplified DNA isolated from tail clippings. Breeding pairs of $\mathrm{B} 6$ recombinaseactivating gene-2-deficient $\left(\right.$ Rag $\left.^{-/-}\right)$mice were purchased from Taconic (Germantown, New York, USA) and maintained by the University of North Carolina Division of Laboratory Animal Medicine in "clean
Table 1

Experimental animal groups

\begin{tabular}{|c|c|c|c|}
\hline Mouse strain & $n$ & Male/Female & Received \\
\hline Rag2-/- & 12 & $5 / 7$ & $1 \times 10^{8}$ anti-MPO cells \\
\hline Rag $^{-/-}$ & 4 & $2 / 2$ & $5 \times 10^{7}$ anti-MPO cells \\
\hline Rag2-/- & 4 & $2 / 2$ & $1 \times 10^{7}$ anti-MPO cells \\
\hline Rag2-/- & 6 & $2 / 4$ & $1 \times 10^{8}$ anti-BSA cells \\
\hline Rag2-/- & 4 & $2 / 2$ & $5 \times 10^{7}$ anti-BSA cells \\
\hline Rag2-/- & 4 & $2 / 2$ & $1 \times 10^{7}$ anti-BSA cells \\
\hline Rag $2^{-/-}$ & 6 & $4 / 2$ & $1 \times 10^{8}$ nonimmunized cells \\
\hline Rag2 $^{-/-}$ & 3 & $2 / 1$ & $5 \times 10^{7}$ nonimmunized cells \\
\hline Rag2-/- & 3 & $2 / 1$ & $1 \times 10^{7}$ nonimmunized cells \\
\hline $\mathrm{Mpo}^{-/-}$ & 4 & $2 / 2$ & $6.5 \times 10^{7}$ anti-MPO cells \\
\hline $\operatorname{Rag}^{-/-}$ & 5 & $0 / 5$ & $50 \mu \mathrm{g} / \mathrm{g}$ anti-MPO IgG \\
\hline Rag2-/- & 3 & $0 / 3$ & $50 \mu \mathrm{g} / \mathrm{g}$ anti-BSA IgG \\
\hline Rag2-/- & 4 & $4 / 0$ & None \\
\hline WT B6 & 6 & $0 / 6$ & $50 \mu \mathrm{g} / \mathrm{g}$ anti-MPO $\operatorname{lgG}$ \\
\hline WT B6 & 3 & $0 / 3$ & $50 \mu \mathrm{g} / \mathrm{g}$ anti-BSA IgG \\
\hline
\end{tabular}

rooms" in autoclaved cages with microisolator tops. Rag2 ${ }^{-/}$mice lack the ability to initiate $V(D) J$ rearrangement and thus do not produce $\mathrm{T}$ or $\mathrm{B}$ lymphocytes with antigen receptors (10). $\mathrm{Mpo}^{-/-}$mice, 8-10 weeks old, were used for immunization and as donors of splenocytes and anti-MPO antibodies. Rag2 ${ }^{-/-}$mice (10-12 weeks old), $\mathrm{MPo}^{-/-}$mice (13 weeks old), and wild-type (WT) B6 mice (9-10 weeks old) were used as recipients for adoptive transfer experiments. Table 1 summarizes the characteristics of the experimental groups. The University of North Carolina Institutional Animal Care and Use Committee approved all animal experiments.

Immunization and detection of anti-MPO. $\mathrm{Mpo}^{-/-}$mice were primed by intraperitoneal injection of $10 \mu \mathrm{g}$ of purified MPO or BSA in complete Freund's adjuvant on day 0 . They were boosted intraperitoneally with the $10 \mu \mathrm{g}$ MPO in incomplete Freund's adjuvant on day 21 and day 36, and boosted by intravenous injection of 10 $\mu \mathrm{g}$ MPO without adjuvant 4 days before splenocytes were harvested. Development of antibodies was monitored by anti-MPO ELISA. Circulating anti-MPO was confirmed in selected animals by indirect immunofluorescence microscopy assay (IFA). For anti-MPO ELISA, microtiter plates were coated with $0.5 \mu \mathrm{g}$ per well murine MPO, incubated with 100 -fold dilutions of mouse sera, developed with alkaline phosphatase-conjugated goat antibodies specific for mouse IgG, and analyzed spectrophotometrically at OD $405 \mathrm{~nm}$. Results were expressed as percentage of a positive control serum pool. For IFA, mouse neutrophils were harvested from the peritoneum of $\mathrm{B} 6$ or $\mathrm{MPO}^{-/-}$mice 4 hours after intraperitoneal injection of $3 \%$ sterilized Proteose peptone (Difco Laboratories, Detroit, Michigan, USA). Isolated cells were washed in PBS with $0.05 \mathrm{mM}$ EDTA and adjusted to $1 \times 10^{6} \mathrm{cells}$ per milliliter. More than $50 \%$ of isolated cells were polymorphonuclear neutrophils, and the remainder were mononuclear leukocytes. Cells were cytocentrifuged onto glass slides, air-dried, and fixed with 
$100 \%$ ethanol for 5 minutes. Serum diluted 1:20 was incubated with the substrate neutrophils for $45 \mathrm{~min}$ utes at room temperature, then washed with PBS. Bound antibody was detected with a 1:50 dilution of FITC-conjugated rabbit anti-mouse IgG (DAKO Corp., Carpinteria, California, USA). Neutrophils from $\mathrm{MpO}^{-/-}$mice were used as negative controls.

Adoptive transfer of splenocytes and Ig. We isolated splenocytes from immunized and control $\mathrm{Mpo}^{-/-}$mice by disrupting the spleens into cold RPMI 1640 medium and then washing twice with RPMI 1640. Red blood cells were removed with lysis buffer (SigmaAldrich, St. Louis, Missouri, USA) followed by washing with RPMI 1640 and final suspension in sterile PBS. Suspensions of $1 \times 10^{7}, 5 \times 10^{7}$, or $1 \times 10^{8}$ splenocytes in $500 \mu \mathrm{l}$ PBS were administered via the tail vein to Rag2 $2^{-/-}$mice $(n=46)$ (Table 1$)$. A group of $\mathrm{Mpo}^{-/-}$mice $(n=4)$ received $6.5 \times 10^{7}$ anti-MPO splenocytes. No differences were observed between males and females in any of the experimental parameters measured.

$\gamma$-Globulins were isolated from serum of $\mathrm{MpO}^{-/-}$mice immunized with MPO or BSA by $50 \%$ ammonium sulfate precipitation. IgG was isolated from the $\gamma$-globulin using HiTrap protein G HP column affinity chromatography (Amersham Pharmacia Biotech). The purified IgG was dialyzed against PBS. Sterile-filtered IgG in PBS was injected via the tail vein at a concentration

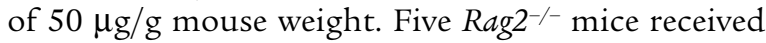
anti-MPO IgG and three received anti-BSA IgG; and six WT B6 mice received anti-MPO IgG and three received anti-BSA IgG (Table 1). Induction of circulating antiMPO (MPO-ANCA) by adoptive transfer of lymphocytes and by passive transfer of IgG was monitored by anti-MPO ELISA and by ANCA IFA.

Laboratory and pathologic evaluation of disease induction. Mice were placed in metabolic cages for 12 hours to collect urine for analysis. Urine was tested by dipstick for hematuria, proteinuria, and pyuria (Roche Diagnostics Corp., Indianapolis, Indiana, USA). Serum creatinine and blood urea nitrogen (BUN) were measured using a Johnson \& Johnson Clinical Diagnostics VITROS 250. Mice were euthanized with methoxyflurane. At the time of postmortem examination, samples of kidney, lung, liver, spleen, and lymph node (axillary or inguinal) were fixed in $10 \%$ formalin and processed for light microscopy. Three B6 mice that received anti-MPO IgG had focal ear ulcers that were sampled for histologic examination. All specimens were stained with hematoxylin and eosin (H\&E), and periodic acid Schiff stains, and selected specimens were stained with Masson trichrome and phosphotungstic acid hematoxylin stains. A sample of each kidney was snap-frozen and processed for direct immunofluorescence microscopy using FITC-labeled antibodies specific for mouse $\operatorname{IgG}, \operatorname{IgA}, \operatorname{IgM}, \mathrm{C} 3$, MPO, and fibrin. Selected kidney specimens were fixed in glutaraldehyde and processed for electron microscopy. Pathologic evaluation was carried out without knowledge of the experimental conditions.

\section{Results}

Development of circulating MPO-ANCA after transfer of antiMPO splenocytes. Rag $2^{-/-}$mice that received anti-MPO splenocytes developed circulating anti-MPO (MPOANCA) within 3 days (Figure 1). The titer continued to rise until sacrifice at 13 days. There was a dose response to anti-MPO splenocytes, with a dose of $1 \times 10^{7}$ splenocytes producing a substantially lower induction of circulating anti-MPO antibodies compared with a dose of $1 \times 10^{8}$ or $5 \times 10^{7}$ splenocytes. Mice that received splenocytes from control mice that had been immunized with anti-BSA or had not been immunized did not develop anti-MPO antibodies. Nonimmunized $\mathrm{Mpo}^{-/-}$mice that received $6.5 \times 10^{7}$ anti-MPO splenocytes had mean anti-MPO ELISA titers of 12.1, 75.6, 90.6, and 98.9 at days $0,3,8$, and 13 , respectively. The presence of anti-MPO antibodies in $\mathrm{Rag}^{-/-}$mice was confirmed by indirect IFA using normal mouse neutrophils as positive substrate and $\mathrm{Mpo}^{-/-}$mouse neutrophils as negative control.

Development of necrotizing and crescentic glomerulonephritis, granulomatous inflammation, and vasculitis after transfer of anti-MPO splenocytes. Rag2 $2^{-/-}$mice that received $1 \times 10^{8}$ or $5 \times 10^{7}$ anti-MPO splenocytes developed severe renal failure that resulted in marked elevation of BUN and serum creatinine (Figure 2 ). In contrast, Rag2 $2^{-/-}$mice that received $1 \times 10^{7}$ anti-MPO splenocytes, or any dose of anti-BSA splenocytes or control splenocytes, developed minimal if any renal insufficiency. Urinalysis revealed that all $R a g 2^{-/-}$mice that received splenocytes developed urine abnormalities (Figure 3). However, $\mathrm{Mpo}^{-/-}$mice that received $6.5 \times 10^{7}$ anti-MPO splenocytes developed no urine abnormalities (data not shown). The urine abnormalities that developed in Rag2-/- mice that received splenocytes were dosedependent in all groups. No effects of sex on these or any other measures of disease induction were observed.

Gross examination revealed hemorrhagic dots on the surface of the kidneys in mice that received $1 \times 10^{8}$ or

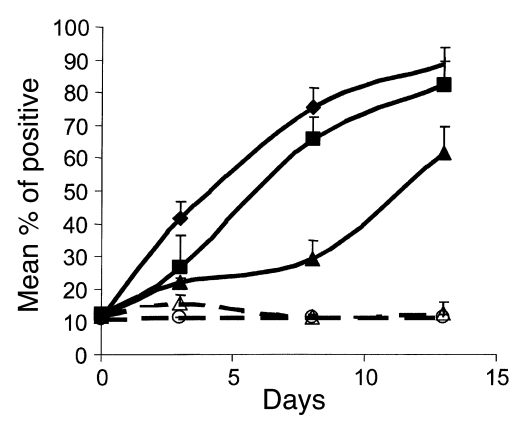

\section{Figure 1}

Anti-MPO antibody ELISA titers in Rag2-/- mice that received $1 \times 10^{8}$ anti-MPO splenocytes (closed diamonds), $5 \times 10^{7}$ anti-MPO splenocytes (filled squares), $1 \times 10^{7}$ anti-MPO splenocytes (filled triangles), $1 \times 10^{8}$ anti-BSA splenocytes (open triangles), or $1 \times 10^{8}$ splenocytes from nonimmunized mice (open circles). Mice that received $5 \times 10^{7}$ or $1 \times 10^{7}$ anti-BSA splenocytes had no values above 15 (data not shown). 


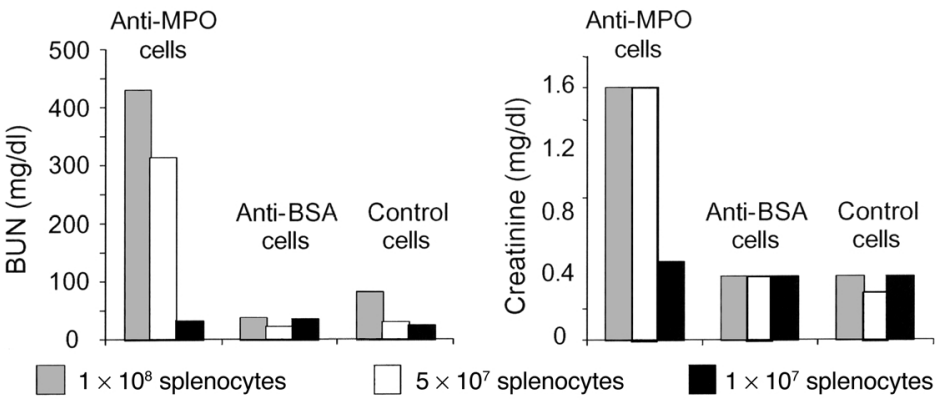

\section{Figure 2}

Mean BUN and serum creatinine in Rag2 ${ }^{-/-}$mice 13 days after they received $1 \times 10^{8}, 5 \times 10^{7}$, or $1 \times 10^{7}$ anti-MPO splenocytes, anti-BSA splenocytes, or nonimmunized control splenocytes. The normal mouse assay reference range was $18-29 \mathrm{mg} / \mathrm{dl}$ for BUN and 0.2-0.8 mg/dl for serum creatinine. Samples taken before injection of splenocytes were within the reference ranges (data not shown).
$5 \times 10^{7}$ anti-MPO splenocytes but not in mice that received other doses of splenocytes. All 16 Rag $2^{-/-}$mice that received $1 \times 10^{8}$ or $5 \times 10^{7}$ anti-MPO splenocytes developed severe necrotizing and crescentic glomerulonephritis (Figure 4; Figure 5, c and d). Crescents involved an average of $83.8 \%$ of glomeruli in mice that received $1 \times 10^{8}$ anti-MPO splenocytes (range 35-99\%) and $85.0 \%$ in mice that received $5 \times 10^{7}$ anti-MPO splenocytes (range 48-99\%) (Figure 4). None of the 30 Rag2 $2^{-/-}$mice that received anti-BSA or control splenocytes or $1 \times 10^{7}$ anti-MPO splenocytes developed glomerular crescents (Figure 4). All Rag2 ${ }^{-/-}$mice that received $1 \times 10^{8}$ or $5 \times 10^{7}$ anti-MPO splenocytes developed segmental or global glomerular necrosis, often in association with crescent formation. Necrosis involved an average of $63.3 \%$ of glomeruli in mice that received $1 \times 10^{8}$ anti-MPO splenocytes (range 33-87\%) and $82.5 \%$ in mice that received $5 \times 10^{7}$ anti-MPO splenocytes (range $42-97 \%$ ). Necrosis was rare in mice that received anti-BSA or control splenocytes or $1 \times 10^{7}$ antiMPO splenocytes (Figure 4).

Most mice that received $1 \times 10^{8}$ or $5 \times 10^{7}$ anti-MPO, anti-BSA, or normal control splenocytes developed mild to moderate glomerular endocapillary hypercellularity that was similar irrespective of the type of splenocytes received (Figures 4 and $5 \mathrm{~b}$ ). Mice that received $1 \times 10^{7}$ splenocytes had minimal or no histologic renal abnormalities (Figure 5a).

Of the 16 Rag $2^{-/-}$mice that received $1 \times 10^{8}$ or $5 \times 10^{7}$ anti-MPO splenocytes, one was found to have necrotizing arteritis in spleen and lymph nodes, one necrotizing arteritis in the lungs, five hemorrhagic pulmonary capillaritis, and one necrotizing granulomatous inflammation in the spleen (Figure 6). The light microscopic appearance of these lesions was identical to that of human ANCA-associated vasculitis, such as microscopic polyangiitis and Wegener granulomatosis. None of the 30 Rag $^{-/-}$mice that received $1 \times 10^{7}$ anti-MPO splenocytes, or any dose of anti-BSA splenocytes or control splenocytes, was found to have vasculitis or granulomatous inflammation.

Immunofluorescence microscopy demonstrated that all $\mathrm{Rag}^{-/-}$mice that received anti-MPO, anti-BSA, or normal control splenocytes developed similar mild to moderate granular glomerular localization of mouse Ig's (Figures 4 and $5 \mathrm{f}$ ), C3, and MPO. In general, the relative intensity of staining was $\mathrm{C} 3>\operatorname{IgG}>\operatorname{IgM}>\operatorname{IgA}>\mathrm{MPO}$. The staining for MPO colocalized with the immune deposits and was accentuated at sites of necrosis. Glomerular staining was more intense in mice that received $1 \times 10^{8}$ or $5 \times 10^{7}$ splenocytes than in those that received $1 \times 10^{7}$ splenocytes. Glomerular staining for MPO was no different in mice that received antiMPO splenocytes compared with those that received anti-BSA or control splenocytes. Only mice that received $1 \times 10^{8}$ or $5 \times 10^{7}$ anti-MPO splenocytes developed marked focally variable glomerular staining for fibrin that corresponded to foci of glomerular necrosis and crescent formation (Figure 5e). No localization of Ig's or complement was identified in renal arteries.

Electron microscopy performed on kidney tissue from four mice that received $1 \times 10^{8}$ or $1 \times 10^{7}$ anti-MPO

\section{Figure 3}

Urinalysis results in Rag2 ${ }^{-/-}$mice 13 days after they received $1 \times 10^{8}, 5 \times 10^{7}$, or $1 \times 10^{7}$ anti-MPO splenocytes, anti-BSA splenocytes, or nonimmunized control splenocytes. Samples taken before injection of splenocytes showed mean proteinuria $1.0+$, hematuria $0.2+$, and leukocyturia $0.0+$ (data not shown).
}
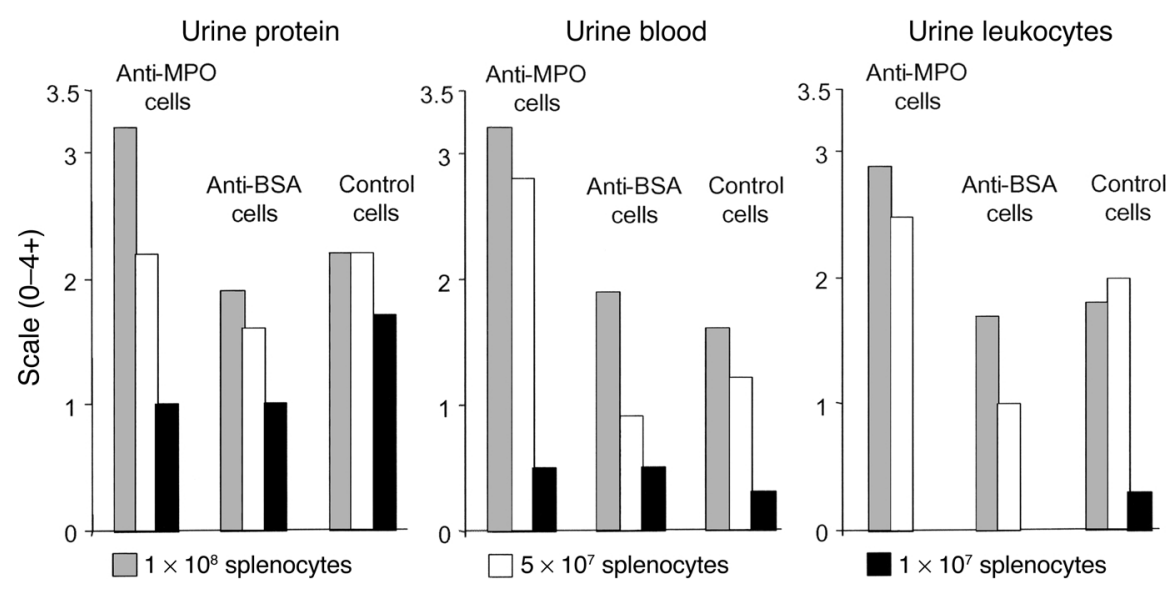

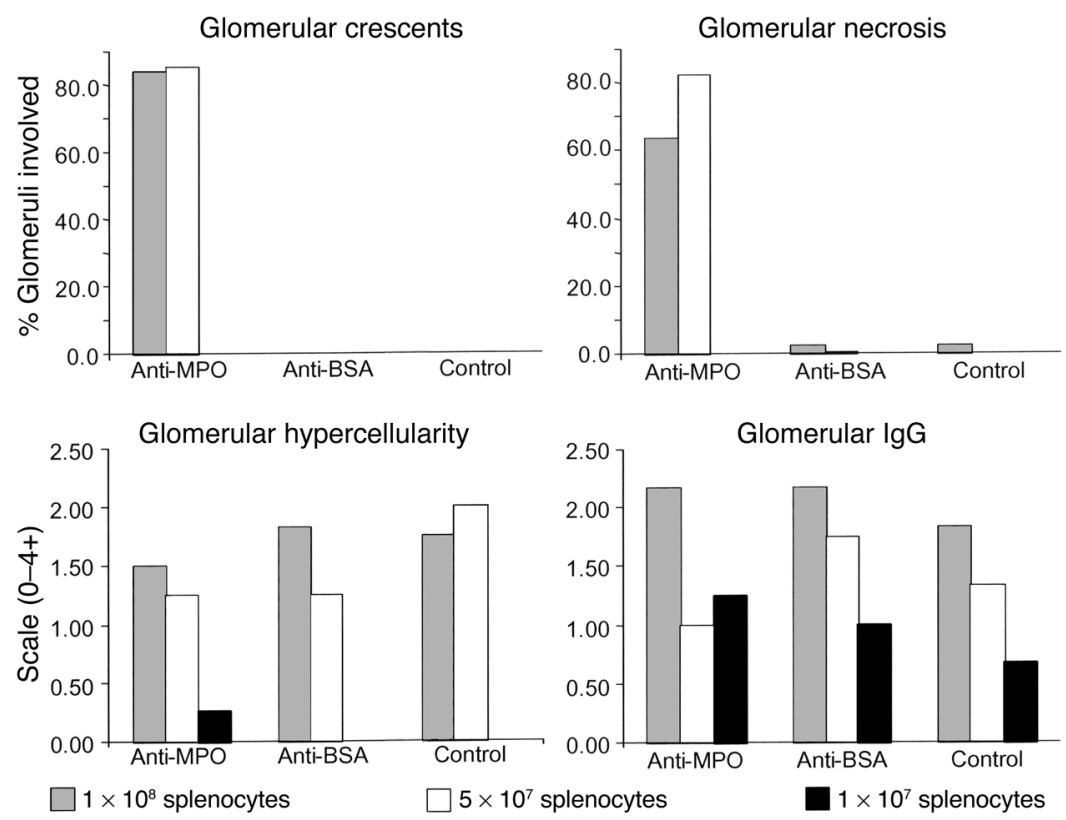

\section{Figure 4}

Pathologic findings in Rag2 ${ }^{-/-}$mice 13 days after they received $1 \times 10^{8}, 5 \times 10^{7}$, or $1 \times 10^{7}$ antiMPO splenocytes, anti-BSA splenocytes, or nonimmunized control splenocytes. The extent of glomerular crescent formation is expressed as the mean percent of glomeruli with crescents in each animal. The extent of glomerular necrosis is expressed as the mean percent of glomeruli with necrosis in each animal. The extent of glomerular endocapillary hypercellularity is expressed as the mean on a scale of 0 (none) to $4+$ (severe). The extent of glomerular immunostaining for $\lg G$ is expressed as the mean on a scale of 0 (none) to 4+ (very intense). Normal Rag2 ${ }^{-1-}$ mice had no crescents, necrosis, endocapillary hypercellularity, or glomerular Ig staining (data not shown). splenocytes and two mice that received $1 \times 10^{8}$ anti-BSA splenocytes demonstrated mesangial immune complex-type electron-dense deposits in all mice and a few subendothelial deposits in one mouse in each group. Areas of segmental necrosis in the mice that received anti-MPO splenocytes had breaks in glomerular basement membranes, fibrin tactoids in capillary lumens and Bowman's space, and cellular crescent formation. Development of circulating MPO-ANCA after passive transfer of anti-MPO antibodies. A single intravenous dose of anti-MPO IgG resulted in an immediate high level of circulating anti-MPO antibodies that subsequently declined. The mean anti-MPO ELISA titer in Rag2 ${ }^{-/-}$ mice was 15.6 prior to injection of anti-MPO IgG, 136.4 one hour after injection, 102.8 after 3 days, and 76.8 after 6 days. Passive transfer of anti-BSA IgG resulted in no increase in anti-MPO reactivity in serum, with mean anti-MPO titers of $15.2,15.5,15.3$, and 15.2 at 0 hours, 1 hour, 3 days, and 6 days, respectively. The mean anti-MPO titer in WT B6 mice that received antiMPO IgG was 109.5 one hour after injection, 100.7 after 3 days, and 98.7 after 6 days. The mean anti-MPO titer in WT B6 mice that received anti-BSA IgG was 16.5 one hour after injection, 15.7 after 3 days, and 15.7 after 6 days. The presence of anti-MPO antibodies was confirmed by indirect immunofluorescence assay on normal mouse neutrophils.

Development of glomerulonephritis after passive transfer of anti-MPO antibodies. By day 3 , mice that received antiMPO IgG already had developed hematuria, proteinuria, and leukocyturia, which persisted until sacrifice at day 6 (Table 2). Mice that received anti-BSA IgG did not develop hematuria, leukocyturia, or proteinuria above background. Rag2 $2^{-/}$mice that received anti-

\section{Figure 5}

Glomerular lesions in Rag2-/- mice 13 days after they received splenocytes. (a) No light microscopic abnormality in a glomerulus from a mouse that received $1 \times 10^{7}$ antiMPO splenocytes. (b) Moderate (2+) endocapillary hypercellularity in a mouse that received $1 \times 10^{8}$ anti-BSA splenocytes. (c) Segmental fibrinoid necrosis (arrow) in a mouse that received $1 \times 10^{8}$ anti-MPO splenocytes. (d) Cellular crescent (arrow) in a mouse that received $1 \times 10^{8}$ anti-MPO splenocytes. (e) Immunofluorescence staining for fibrin in a crescent in a mouse that received $1 \times 10^{8}$ anti-MPO splenocytes. (f) Predominantly mesangial moderate $(2+)$ immunofluorescence staining for $\lg G$ in a mouse that received $1 \times 10^{8}$ anti-BSA splenocytes. Periodic acid Schiff stain for light microscopy is shown.

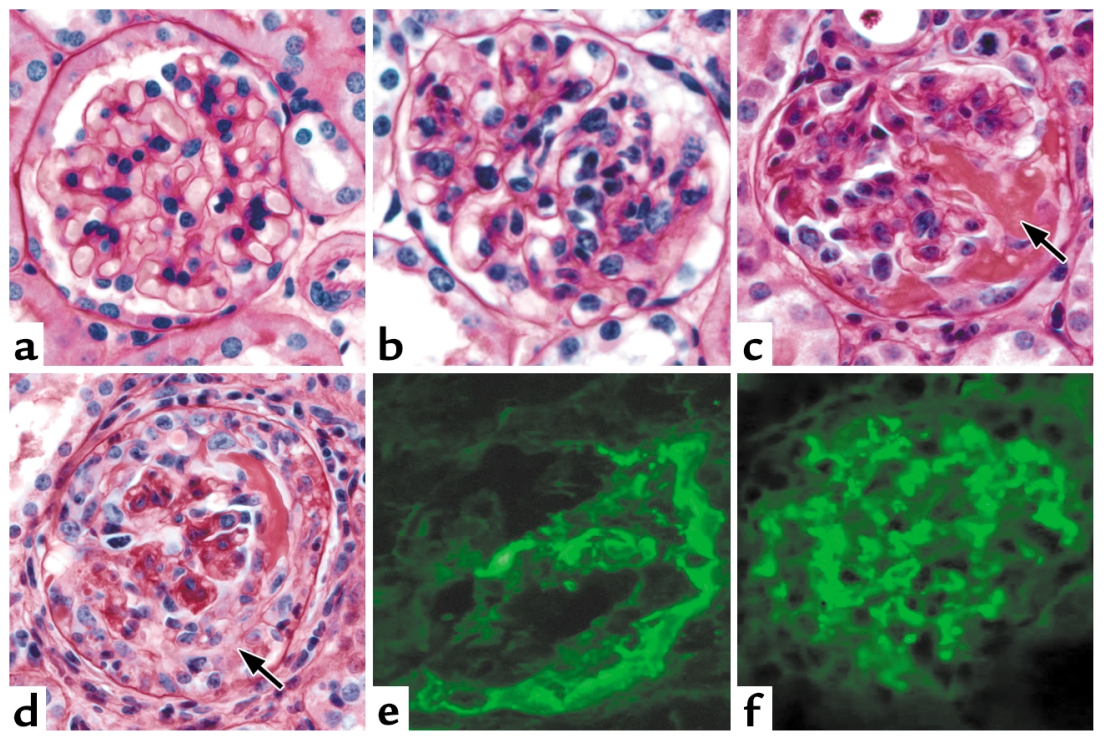




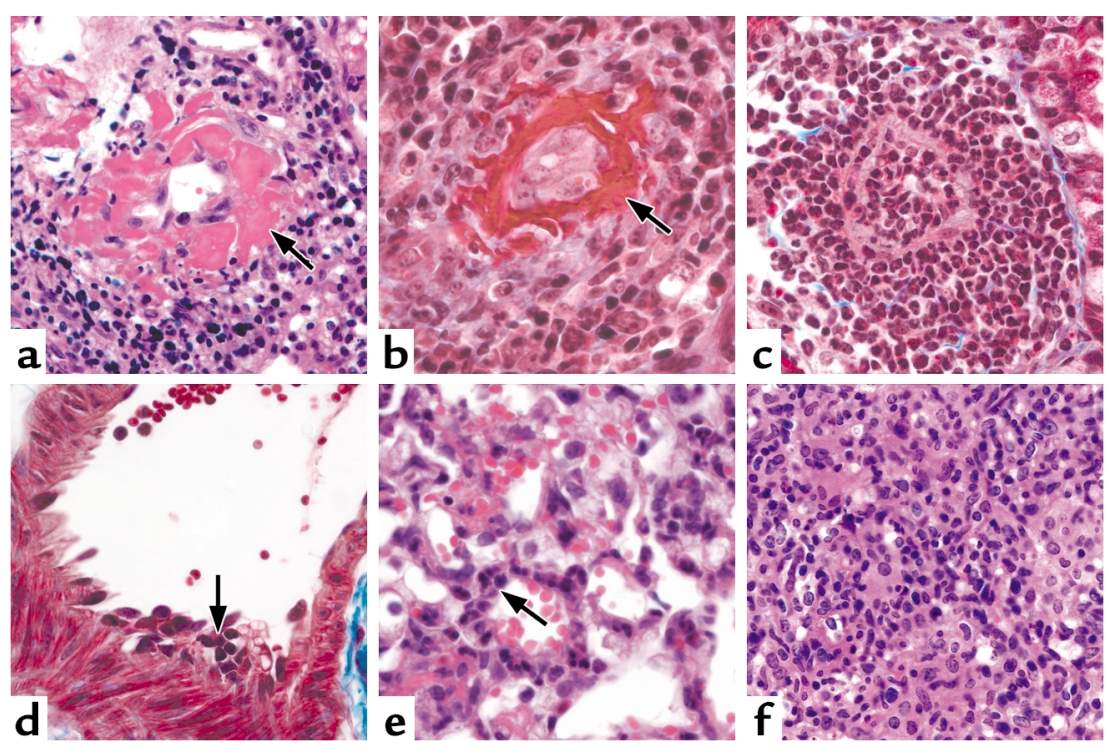

\section{Figure 6}

Systemic vasculitis and granulomatous inflammation in Rag2-/- mice 13 days after receiving $1 \times 10^{8}$ anti-MPO splenocytes. (a) Necrotizing arteritis in lymph node with transmural fibrinoid necrosis (arrow) (H\&E stain). (b) Necrotizing arteritis in spleen with transmural fibrinoid necrosis (arrow) (Masson trichrome stain). (c) Intense acute arteritis in lung with transmural and perivascular infiltration of predominantly neutrophils (Masson trichrome stain). (d) Early focal arteritis in lung with neutrophils and mononuclear leukocytes invading the intima (arrow) (Masson trichrome stain). (e) Pulmonary hemorrhagic capillaritis showing numerous neutrophils marginated within alveolar septal capillaries (arrow) and red blood cells in air spaces. (f) Granulomatous inflammation with multinucleated giant cells in a lymph node.
MPO IgG, which had the most severe renal lesions pathologically, were the only group to have slightly elevated BUN (Table 2). Serum creatinine did not differ between mice that received anti-MPO $\operatorname{IgG}(0.3 \mathrm{mg} / \mathrm{dl})$ and those that received anti-BSA $\operatorname{IgG}(0.3 \mathrm{mg} / \mathrm{dl})$.

All five $R a g 2^{-/-}$mice sacrificed 6 days after receiving anti-MPO IgG had focal necrotizing glomerulonephritis (mean $13.2 \%$ of glomeruli with necrosis) and crescents (mean $10.8 \%$ of glomeruli with crescents), whereas mice that received anti-BSA IgG had no histologic lesions (Table 2; Figure 7). Likewise, all six WT B6 mice sacrificed 6 days after receiving anti-MPO $\operatorname{IgG}$ had focal necrotizing glomerulonephritis (mean $4.7 \%$ of glomeruli with necrosis) and crescents (mean $3.3 \%$ of glomeruli with crescents), whereas WT B6 mice that received antiBSA IgG had no histologic lesions (Table 2; Figure 8). Electron microscopy revealed no immune complex-type electron-dense deposits in the glomeruli of the five $\mathrm{Rag}^{-/-}$mice that received anti-MPO IgG. Immunofluorescence microscopy demonstrated little or no glomerular staining for Ig's, C3, or MPO in Rag2-/- and WT B6 mice that received anti-MPO IgG. There was trace mesangial staining for Ig's that was no different between mice that received anti-MPO or anti-BSA IgG. There was slightly increased focal segmental staining predominantly for IgG and C3 at sites of necrotizing injury (Figure $7 \mathrm{f}$ ). There was trace staining at these sites for MPO.
Mice that received anti-BSA IgG lacked the focal segmental glomerular staining for IgG, C3, and MPO. Mice that received anti-MPO IgG had intense focal segmental glomerular staining for fibrin that corresponded to foci of segmental necrosis and crescent formation (Figures $7 e$ and $8 d$ ). There was no staining for fibrin in glomeruli of mice that received anti-BSA IgG. The paucity of staining for Ig's and complement in the glomeruli of mice with glomerulonephritis induced by anti-MPO IgG was identical to the pattern of staining seen with human ANCA-associated pauci-immune glomerulonephritis.

Focal pulmonary alveolar capillaritis was identified in two of the six WT B6 mice that received anti-MPO IgG (Figure 8f). Three of the WT B6 mice that received antiMPO IgG had grossly discernible cutaneous lesions on the ears. Histologically, all three had focal ulceration and infarction. Necrotizing arteritis with fibrinoid necrosis and leukocytoclasia was identified in one specimen (Figure 8e). The vasculitic lesions caused by anti-MPO IgG in the WT B6 mice were histologically identical to alveolar capillaritis and necrotizing arteritis in humans with ANCA-associated vasculitis.

\section{Discussion}

This experimental model provides convincing evidence that anti-MPO antibodies cause crescentic glomerulonephritis and small-vessel vasculitis. All 16

Table 2

Renal abnormalities in Rag2-/- and WT B6 mice 6 days after they received anti-MPO or anti-BSA IgG

\begin{tabular}{|c|c|c|c|c|}
\hline Type of IgG and type of mouse & $\begin{array}{c}\% \text { Crescents } \\
\text { (mean \& range) }\end{array}$ & $\begin{array}{c}\% \text { Necrosis } \\
\text { (mean \& range) }\end{array}$ & $\begin{array}{l}\text { BUN (mg/dl) } \\
\text { (mean \& range) }\end{array}$ & $\begin{array}{c}\text { Prot/Hem/Leu } \\
(0-4+)\end{array}$ \\
\hline Anti-MPO IgG in Rag2-/- mice & $10.8 \%(5-15)$ & $13.2 \%(11-24)$ & $47.4(34-54)$ & $2.0 / 2.7 / 1.8$ \\
\hline 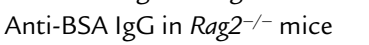 & $0 \%$ & $0 \%$ & $22.7(21-25)$ & $1.0 / 0.5 / 0.0$ \\
\hline No IgG in Rag2 $2^{-/-}$mice & $0 \%$ & $0 \%$ & $21.4(19-31)$ & $1.0 / 0.0 / 0.0$ \\
\hline Anti-MPO IgG in WT B6 mice & $3.3 \%(2-6)$ & $4.7 \%(3-7)$ & $23.3(21-27)$ & $1.6 / 2.2 / 1.2$ \\
\hline Anti-BSA IgG in WT B6 mice & $0 \%$ & $0 \%$ & $25.7(22-29)$ & $1.0 / 0.0 / 0.0$ \\
\hline
\end{tabular}

Prot/Hem/Leu, proteinuria/hematuria/leukocyturia. 

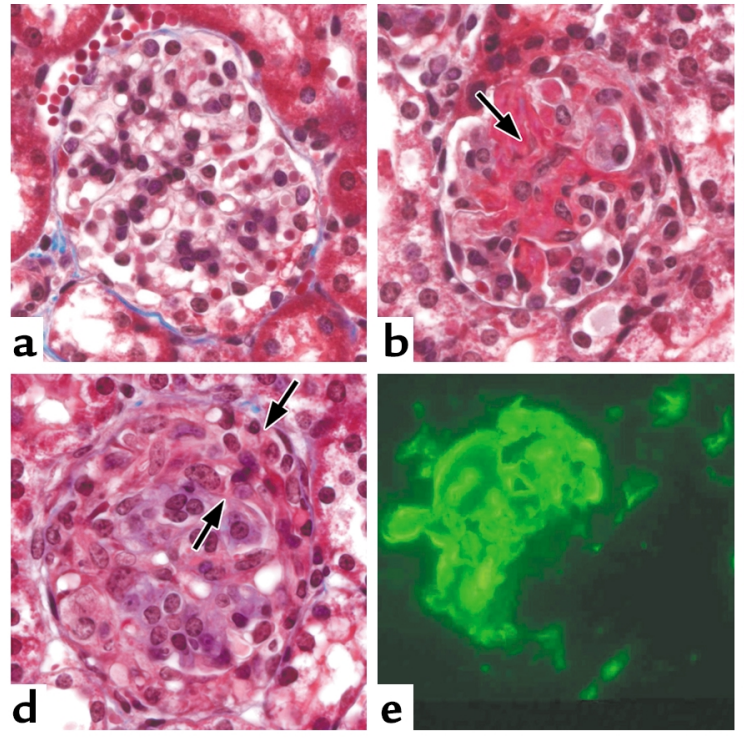

\section{Figure 7}

Glomerular lesions in Rag2-/- mice 6 days after receiving anti-MPO IgG. (a) Glomerulus with no lesion. (b) Segmental fibrinoid necrosis (arrow). (c) Segmental fibrinoid necrosis with an adjacent small cellular crescent (arrow). (d) Large circumferential cellular crescent (between arrows) completely surrounding a glomerulus. (e) Immunofluorescence microscopy for fibrin showing prominent staining corresponding to segmental necrosis and crescent formation. (f) Immunofluorescence microscopy for IgG showing a paucity of segmental staining corresponding to an area of segmental necrosis. Masson trichrome staining for light microscopy is shown.
Rag2 $^{-/-}$mice that received $5 \times 10^{7}$ or $1 \times 10^{8}$ anti-MPO splenocytes and all five Rag2 ${ }^{-/}$mice and all six WT B6 mice that received $50 \mu \mathrm{g} / \mathrm{g}$ anti-MPO IgG developed glomerular crescents and necrosis. None of 17 Rag2mice that received anti-BSA splenocytes or anti-BSA IgG developed glomerular crescents, nor did any of the mice that received only $1 \times 10^{7}$ anti-MPO splenocytes. None of three WT B6 mice that received antiBSA IgG developed crescents.

All Rag2 $2^{-/-}$mice that received splenocytes from immune-competent $\mathrm{Mpo}^{-/-}$mice developed low to moderate levels of glomerular immune complex localization. These immune deposits caused mild to moderate proliferative glomerulonephritis but, in the absence of antiMPO, caused little or no renal insufficiency and no glomerular crescents or necrosis. The basis for the uniform production of glomerular immune deposits is not known but probably relates to the introduction of functioning lymphocytes into mice that previously had no functioning adaptive immune system. This low to moderate level of glomerular immune complex localization was the same in intensity and composition in mice irrespective of the transfer of anti-MPO, anti-BSA, or control splenocytes. The basis for this immune complex glomerulonephritis is unknown. Possibilities include reactions of the newly synthesized antibodies with circulating exogenous antigens, or production of autoantibodies possibly as a component of a graft-versus-host reaction. The latter is not likely, since all of the mice had the same B6 background and thus shared the same major histocompatibility antigens. Induction of an autoimmune response remains a possibility; however, preliminary cell extract immunoprecipitation analysis of serum from the mice that received splenocytes, performed by Hanno Richards at the University of Florida (Gainesville, Florida, USA), revealed no autoantibodies, including no antibodies against DNA, Sm, or ribonucleoproteins (data not shown).

\section{Figure 8}

Vasculitic lesions in WT B6 mice 6 days after they received anti-MPO IgG. (a) Glomerulus with segmental fibrinoid neurosis (periodic acid Schiff stain). (b) Glomerulus with segmental fibrinoid necrosis and crescent formation (periodic acid Schiff stain). (c) Glomerulus with segmental fibrinoid necrosis and crescent formation (H\&E stain). (d) Immunofluorescence microscopy for fibrin showing prominent staining corresponding to segmental necrosis and crescent formation. (e) Necrotizing arteritis with leukocytoclasia in the dermis of the ear (H\&E stain). (f) Pulmonary alveolar capillaritis on the left and more normal lung on the right.
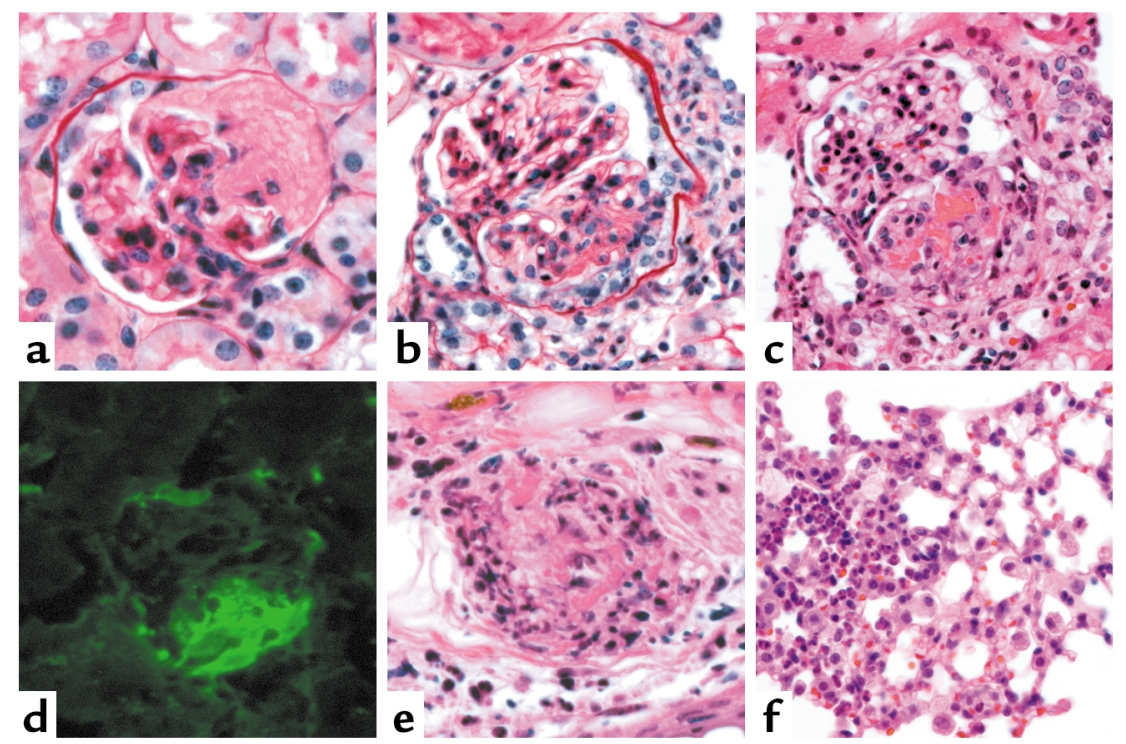
In striking contrast to Rag2 $2^{-/-}$mice that received antiBSA or normal control splenocytes, mice that received anti-MPO splenocytes developed very severe necrotizing and crescentic glomerulonephritis and small-vessel vasculitis. This disease induction was dose-dependent, since all mice that received $5 \times 10^{7}$ or $1 \times 10^{8}$ anti-MPO splenocytes developed severe crescentic glomerulonephritis but those that received $1 \times 10^{7}$ developed no crescents at all. This dose effect is in accord with the serum titers of anti-MPO attained after the different doses of splenocytes, which were similarly high in mice that received $5 \times 10^{7}$ or $1 \times 10^{8}$ anti-MPO splenocytes and substantially lower in those that received $1 \times 10^{7}$ anti-MPO splenocytes (Figure 1).

In addition to necrotizing and crescentic glomerulonephritis, mice that received anti-MPO splenocytes developed pulmonary hemorrhagic capillaritis and systemic necrotizing arteritis. The lesions were histologically identical to the pulmonary hemorrhagic capillaritis and systemic necrotizing arteritis that occurs in patients with ANCA-associated small-vessel vasculitis, such as microscopic polyangiitis and Wegener granulomatosis $(1,2)$. One mouse even had necrotizing granulomatous inflammation in a lymph node that resembled the granulomatous inflammation of Wegener granulomatosis. Only two or three levels of section of each organ specimen were evaluated for this study. Given the focal nature of the vasculitic lesions and granulomatous inflammation, many more lesions should be detected by evaluating additional tissue sections.

The transfer of splenocytes introduced both antiMPO B lymphocytes and anti-MPO T lymphocytes into recipient mice. Thus, either MPO-specific T lymphocytes or antibodies produced by MPO-specific B lymphocytes could be mediating the glomerulonephritis and vasculitis in the mice that received splenocytes. To evaluate the pathogenicity of anti-MPO antibodies alone (i.e., MPO-ANCAs), purified anti-MPO IgG or anti-BSA IgG was injected into Rag2 ${ }^{-/-}$mice and WT B6

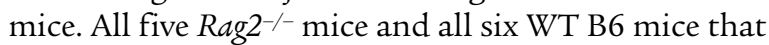
received anti-MPO IgG developed focal glomerular necrosis and crescent formation, whereas none of the six mice that received anti-BSA IgG did. This demonstrates that anti-MPO IgG causes glomerular necrosis and crescents in the absence of antigen-specific $T$ and

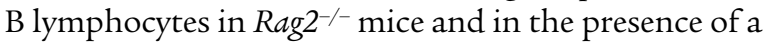
competent immune system in WT B6 mice. The glomerular lesions that were caused in mice by antiMPO IgG were identical by light microscopy and immunofluorescence microscopy to the glomerular lesions of human ANCA-associated pauci-immune glomerulonephritis and small-vessel vasculitis $(1,2)$. Both mouse and human glomerular lesions have fibrinoid necrosis, crescent formation, and an absence or paucity of staining for Ig's by immunofluorescence microscopy. The WT B6 mice also developed vasculitic lesions identical to human ANCA-associated disease in other organs, including pulmonary alveolar capillaritis and cutaneous necrotizing arteritis.
The so-called pauci-immune characteristic of ANCAassociated glomerulonephritis is pathologically very distinct from the substantial vessel wall localization of Ig's in immune complex-mediated glomerulonephritis and glomerulonephritis induced by anti-glomerular basement membrane (anti-GBM) antibody. This suggests that the pathogenesis of pauci-immune ANCAassociated glomerulonephritis is distinct from that for immune complex glomerulonephritis or anti-GBM glomerulonephritis. This distinct mechanism appears to involve ANCA-induced activation of neutrophils and monocytes. ANCA IgG can activate neutrophils and monocytes in vitro $(5,6)$. For example, ANCA IgG stimulates cytokine-primed neutrophils to release injurious oxygen metabolites and proteinases (11-13). Activation of neutrophils by ANCA IgG also induces the release of numerous proinflammatory cytokines, such as IL-1, IL-8, and leukotrienes (14-16). Monocytes also have ANCA antigens, including MPO and PR3, and can be induced to undergo a respiratory burst and release of proteinases and cytokines after stimulation by ANCA IgG (17-19). Neutrophils that have been activated by ANCA IgG are capable of adhering to and killing endothelial cells in vitro $(20,21)$.

In vitro and in vivo experiments indicate that the activation of neutrophils and monocytes by ANCA and the induction of tissue injury by ANCA are facilitated by minor inflammatory stimuli that prime leukocytes to interact with ANCA (5-7). This priming causes increased expression of ANCA antigens, such as MPO, at the surface of neutrophils and monocytes where the antigens can interact with ANCA and cause leukocyte activation. The greater severity of the glomerulonephritis induced by anti-MPO splenocytes compared with that induced by anti-MPO IgG might be due to the synergistic presence of the glomerular immune complexes in the former acting as a priming factor for leukocytes. An alternative explanation is that anti-MPO T lymphocytes that are transferred into the recipients with the splenocytes might have an additive effect on the severity of the vascular inflammatory injury.

The in vitro data that document the ability of ANCA IgG to activate neutrophils and monocytes, combined with the current mouse model of ANCA-induced glomerulonephritis and vasculitis, strongly support a primary pathogenic role for ANCA in ANCA-associated glomerulonephritis and vasculitis. Using the Bradford Hill criteria for causation (22), the clinical and experimental evidence that is now available strongly indicates that ANCAs cause ANCA-associated glomerulonephritis and small-vessel vasculitis. The Bradford Hill criteria for concluding that an association is indicative of causation are: 1 , strength; 2 , specificity; 3 , consistency; 4 , temporality; 5, biological gradient; 6 , experimental evidence; 7 , coherence; and 8, analogy.

Criteria 1 and 2 are fulfilled by the very strong and specific clinical association between ANCAs and pauciimmune crescentic glomerulonephritis and small-vessel vasculitis $(1,2)$. Approximately $80-90 \%$ of active 
untreated patients with pauci-immune necrotizing and crescentic glomerulonephritis and vasculitis have circulating MPO-ANCA or PR3-ANCA. Less than 10\% of patients with other types of glomerulonephritis and vasculitis have ANCA, and less than $1 \%$ of the general population has ANCA.

Criterion 3 is fulfilled by the virtually complete consistency among research groups throughout the world in demonstrating the association between ANCAs and pauci-immune glomerulonephritis and small-vessel vasculitis.

Criteria 4, 5, and 6 have been difficult to document clinically, but the experimental data in this article show that crescentic glomerulonephritis and small-vessel vasculitis develop within a few days after introduction of ANCA into experimental animals (temporality), and that the occurrence and magnitude of this effect is dose-dependent (gradient).

Criterion 7 is fulfilled by the coherence of the in vitro observations that ANCA IgG causes activation of neutrophils and monocytes with the in vivo pathologic observations that the acute phase of injury is characterized by necrotizing acute inflammation that is mediated by activated neutrophils and monocytes.

Criterion 8 is fulfilled by the analogy between MPO and PR3, which are the two major antigenic targets of ANCAs. Although they are very different molecules with very different biological function, MPO and PR3 have the same locations in the cytoplasm of neutrophils and monocytes, are similarly displayed and released during varying phases of activation, and are both targeted by ANCAs. These analogies between MPO and PR3 support the pathogenic potential not only of MPO-ANCA but also of PR3-ANCA.

Thus, based on the experimental model described here, we conclude that the association in patients between ANCAs and pauci-immune glomerulonephritis and vasculitis is most likely due to causation. Therefore, we propose that the often-used terms "ANCA-associated glomerulonephritis" and "ANCAassociated vasculitis" should be changed to "ANCA glomerulonephritis" and "ANCA vasculitis." The results of these studies suggest that therapeutic strategies that selectively eliminate or neutralize ANCAs could be effective in treating ANCA glomerulonephritis and vasculitis.

\section{Acknowledgments}

We thank Jue Yao for excellent technical support, Susan Hogan for excellent advice about data presentation, Victoria Madden for expert assistance with photographic reproduction, and Hanno Richards for serologic testing for autoantibodies. This study was supported by NIH grant NIADDK PO1 DK58335 and by internal funding from the University of North Carolina Nephropathology Laboratory. P. Heeringa was supported by grant PC115 from the Dutch Kidney Foundation.

1. Falk, R.J., and Jennette, J.C. 1997. ANCA small-vessel vasculitis. J. Am. Soc. Nephrol. 8:314-322.

2. Jennette, J.C., and Falk, R.J. 1997. Small vessel vasculitis. N. Engl. J. Med. 337:1512-1523.

3. Falk, R.J., and Jennette, J.C. 1988. Anti-neutrophil cytoplasmic autoantibodies with specificity for myeloperoxidase in patients with systemic vasculitis and idiopathic necrotizing and crescentic glomerulonephritis. N. Engl.J. Med. 318:1651-1657.

4. Jennette, J.C., Hoidal, J.H., and Falk, R.J. 1990. Specificity of anti-neutrophil cytoplasmic autoantibodies for proteinase 3. Blood. 75:2263-2264.

5.Jennette, J.C., and Falk, R.J. 1998. Pathogenesis of the vascular and glomerular damage in ANCA-positive vasculitis. Nephrol. Dial. Transplant. 13(Suppl. 1):16-20.

6. Savage, C.O., Harper, L., and Holland, M. 2002. New findings in pathogenesis of antineutrophil cytoplasm antibody-associated vasculitis. Curr. Opin. Rheumatol. 14:15-22.

7. Heeringa, P., Brouwer, E., Cohen Tervaert, J.W., Weening, J.J., and Kallenberg, C.G. 1998. Animal models of anti-neutrophil cytoplasmic antibody associated vasculitis. Kidney Int. 53:253-263.

8. Hope, H.R., et al. 2000. Large-scale purification of myeloperoxidase from HL60 promyelocytic cells: characterization and comparison to human neutrophil myeloperoxidase. Protein Expr. Purif. 18:269-276.

9. Aratani, Y., et al. 1999. Severe impairment in early host defense against Candida albicans in mice deficient in myeloperoxidase. Infect. Immun. 67:1828-1836.

10. Shinkai, Y., et al. 1992. RAG-2-deficient mice lack mature lymphocytes owing to inability to initiate V(D)J rearrangement. Cell. 68:855-867.

11. Falk, R.J., Terrell, R.S., Charles, L.A., and Jennette, J.C. 1990. Anti-neutrophil cytoplasmic autoantibodies induce neutrophils to degranulate and produce oxygen radicals in vitro. Proc. Natl. Acad. Sci. USA. 87:4115-4119.

12. Charles, L.A., Caldas, M.L.R., Falk, R.J., Terrell, R.S., and Jennette, J.C. 1991. Antibodies against granule proteins activate neutrophils in vitro. J. Leukoc. Biol. 50:539-546.

13. Brouwer, E., et al. 1994. Neutrophil activation in vitro and in vivo in Wegener's granulomatosis. Kidney Int. 45:1120-1131.

14. Grimminger, F., et al. 1996. Neutrophil activation by anti-proteinase 3 antibodies in Wegener's granulomatosis: role of exogenous arachidonic acid and leukotriene B4 generation. J. Exp. Med. 184:1567-1572.

15. Brooks, C.J., et al. 1996. IL-1 beta production by human polymorphonuclear leucocytes stimulated by anti-neutrophil cytoplasmic autoantibodies: relevance to systemic vasculitis. Clin. Exp. Immunol. 106:273-279.

16. Cockwell, P., Brooks, C.J., Adu, D., and Savage, C.O. 1999. Interleukin-8: a pathogenetic role in antineutrophil cytoplasmic autoantibody-associated glomerulonephritis. Kidney Int. 55:852-863.

17. Charles, L.A., Falk, R.J., and Jennette, J.C. 1992. Reactivity of antineutrophil cytoplasmic autoantibodies with mononuclear phagocytes. J. Leukoc. Biol. 51:65-68.

18. Casselman, B.L., Kilgore, K.S., Miller, B.F., and Warren, J.S. 1995. Antibodies to neutrophil cytoplasmic antigens induce monocyte chemoattractant protein-1 secretion from human monocytes. J. Lab. Clin. Med. 126:495-502.

19. Ralston, D.R., Marsh, C.B., Lowe, M.P., and Wewers, M.D. 1997. Antineutrophil cytoplasmic antibodies induce monocyte IL-8 release. Role of surface proteinase-3, alpha1-antitrypsin, and Fcgamma receptors. J. Clin. Invest. 100:1416-1424.

20. Ewert, B.H., Jennette, J.C., and Falk, R.J. 1992. Anti-myeloperoxidase antibodies stimulate neutrophils to damage human endothelial cells. Kidney Int. 41:375-383.

21. Savage, C.O., Pottinger, B.E., Gaskin, G., Pusey, C.D., and Pearson, J.D. 1992. Autoantibodies developing to myeloperoxidase and proteinase 3 in systemic vasculitis stimulate neutrophil cytotoxicity toward cultured endothelial cells. Am. J. Pathol. 141:335-342.

22. Hill, A.B. 1965. The environment and disease: association or causation? Proc. R. Soc. Med. 58:295-300. 